\title{
Effect of Epoch Length on Compressed Sensing of EEG
}

\author{
Xue Jun Li, Phuong Thi Dao and Anthony Griffin
}

\begin{abstract}
Aging populations are stretching existing healthcare systems to their limits in both developing and developed countries. Telemedicine is a promising solution to this challenging problem. Under the conventional data compression paradigm, long-time recording of electroencephalography (EEG) signals still generates excessive amount of data, which requires large data storage and long transmission time. While promoting mobile telemedicine with compressed sensing (CS) as a key system for EEG monitoring, this paper investigates the effect of epoch length on CS to compress EEG signals. Experimental results show that a longer epoch length leads to better signal compression at the expense of larger signal reconstruction time. At a sampling frequency of $256 \mathrm{~Hz}$, a 4-s epoch length is suitable when using a general desktop computer to perform signal reconstruction.
\end{abstract}

\section{INTRODUCTION}

The Global Health and Aging report by the World Health Organization (WHO) highlighted that "the number of people aged 65 or older is projected to grow from an estimated 524 million in 2010 to nearly 1.5 billion in 2050 , with most of the increase in developing countries". This implies a pressing need to improve the healthcare availability and affordability in those underdeveloped countries. Moreover, the aging population with increased life expectancy also presents significant challenges to existing health care systems in most developed nations. These challenges may include: (i) increased need for health care resources, (ii) shortage of health care professionals, and (iii) questionable sustainability of current health care systems. It is not easy to overcome these challenges, especially during a downturn of the global economy. Fortunately, technological advances in embedded electronics and software design have paved the way for the deployment of telemedicine [1], which is known as a broad description of infrastructure to provide medical and healthcare service using information and communication technologies (ICT). As a technology-rich alternative to a regular in-person physician visit, telemedicine uses a number of sensor nodes to collect biomedical signals, such as electrocardiogram (ECG) and electroencephalogram (EEG). It will send data through the Internet to a computer operated by a doctor in a hospital. As a result, a patient can receive a professional diagnosis while remaining at home. This paradigm will not only substantially improve availability of medical services, but also reduce their related costs, producing a better medical solution for patients. Noteworthily, recent development of wireless body area networks (WBANs) will expedite the design and development of mobile telemedicine [1], further reducing the time and space barrier between patients and health professionals.

Although telemedicine provides salient benefits as compared conventional health monitoring systems, there are

Xue Jun Li, Phuong Thi Dao and Anthony Griffin are with Department of Electrical and Electronic Engineering, Auckland University of Technology, Auckland 1010, New Zealand \{xuejun.li, pdao, anthony.griffin\}@aut.ac.nz. several major obstacles to realize such a system: (i) The limited energy supplied by on-board batteries requires WBANs to have a low power consumption. (ii) The tremendous amount of sensing data requires efficient data compression techniques to save storage space and shorten the transmission time. (iii) Data compression algorithms at sensor nodes should be computationally light to reduce power consumption and hardware cost [2]. All these factors prevent conventional data compression techniques from being adopted in telemedicine without fundamental changes.

Contrary to conventional data compression techniques, compressed sensing (CS) [3] was proposed to compress the data during sampling, as if it were possible to directly acquire just the important information about the signals. CS significantly reduces the sampling rate as compared to the Nyquist sampling theorem. Furthermore, it does not require a priori knowledge of the signal to be acquired, except that the signal is compressible. This makes CS suitable for telemedicine. A framework of CS for EEG compression and reconstruction was proposed in [4]. It was shown by an empirical study that EEG signals are sparse in a Gabor frame. However, the results presented in [4] are limited to repeated EEG recording trials, such as asking a patient to repeat the same task while recording the EEG signals. Abdulghani et al. performed a feasibility study of CS in compressing EEG signals for a telemedicine system [5]. Later in [6], they studied the performance of CS using different sparsifying bases and reconstruction algorithms, and concluded that the lossy compression of single-channel EEG using CS is applicable only for certain applications with a relaxed tolerance on reconstruction errors. However, they did not study the effect of the length of EEG epochs [7] [8], which are segments of the continuous EEG signal.

Fauvel and Ward presented an energy efficient CS-based compression framework using a Gabor dictionary [9]. They showed the proposed solution using CS achieved eight times more energy efficiency than the typical wavelet based compression method [10]. In their study, the epoch length was arbitrarily chosen as four seconds with a sampling frequency $\left(f_{s}\right)$ of $128 \mathrm{~Hz}$, which was downsampled from Physionet [11] with an original $f_{s}$ of $256 \mathrm{~Hz}$. However, they did not study the effect of the length of EEG epoch. Compression of multichannel EEG using the simultaneous cosparsity and low-rank optimization was studied in [12]. However, the effect of EEG epoch length was not investigated.

\section{RELATED WORK}

EEG is widely used in hospitals and clinics. A specialized technician usually performs the EEG test, and then passes the recorded EEG to a doctor for further analysis. They can be used to predict and diagnose brain diseases and to facilitate the setup of a brain-computer interface (BCI) [13]. For the former, EEG signals are analyzed to identify whether certain 
discovered disease-related patterns appear in the recorded data (i.e., EEG is used to confirm or rule out potential brain disorders such as seizure/epilepsy and head injury); for the latter, EEG signal patterns are associated with a certain task performed by the subject in order to operate a device, such as a wheel chair. Modern hardware and software ease the EEG recording, nevertheless, the data size remains extremely large for long-time multi-channel EEG signal recording.

EEG signal is normally recorded using a 10-20 international system, which can be extended by adding electrodes to produce 32, 64, 128 and even 256 channels [14]. Each electrode is connected to one input of a differential amplifier with a voltage gain of $60 \sim 100 \mathrm{~dB}$. Modern EEG recording systems have an $f_{s}$ of 256 to $512 \mathrm{~Hz}$. Next, long-term (up to several months) recording of EEG is required especially in the diagnosis of certain brain disorders, such as epilepsy [15]. Consequently, the volume of the recorded EEG data can easily exceed 10 gigabytes (GB) per day for a single patient, which are not suitable for storage, real-time monitoring or telemedicine. It is imperative to reduce the data size while preserving their clinical information for diagnosis. As the result, EEG compression methods have been widely studied.

CS is also known as compressive sensing, compressive sampling or sparse sampling. It can efficiently acquire a signal with less samples as compared to the conventional Nyquist sampling, and reconstruct the signal by finding solutions to underdetermined linear systems [3]. Consider a discrete time signal $\mathbf{x}$ in $\mathbb{R}^{\mathrm{N}}$, which can be expressed as

$$
\mathbf{x}=\boldsymbol{\Psi} \mathbf{s}
$$

where $\Psi$ is the $N \times N$ basis matrix (also known as sparsifying dictionary) and $\mathbf{s}$ is the $N \times 1$ weighting coefficients vector, respectively. As such, $\mathbf{x}$ and $\mathbf{s}$ are equivalent representations of the signal - the former is in the time/space domain and the latter in the $\boldsymbol{\Psi}$ domain. If only $K$ of $N$ elements in $\mathbf{s}$ are non-zero (the remaining $N$ - $K$ coefficients are zero), the signal $\mathbf{x}$ is $K$-sparse. Furthermore, if those $N$ elements in $\mathbf{s}$ have a few large values and many small values, the signal $\mathbf{x}$ is compressible.

Suppose $\mathbf{y}$ is a measurement in $\mathbb{R}^{\mathrm{M}}$ of $\mathbf{x}$ using a $M \times N$ sensing matrix (also known as the measurement matrix) $\boldsymbol{\Phi}$, and then we have

$$
\mathbf{y}=\boldsymbol{\Phi} \mathbf{x}=\boldsymbol{\Phi} \Psi \mathbf{s}=\Theta \mathbf{s}
$$

where $\boldsymbol{\Theta}=\boldsymbol{\Phi} \Psi$ is the $M \times N$ transformation matrix.

The CS problem comprises of two sub-problems: (i) design of a stable sensing matrix such that the salient information of $\mathbf{x}$ is not damaged during the data acquisition processing; and (ii) design of a reconstruction algorithm to recover an estimate of $\mathbf{x}, \hat{\mathbf{x}}$ from the measurement $\mathbf{y}$ such that the difference between $\hat{\mathbf{x}}$ and $\mathbf{x}$ is minimized.

Proper design of the $M \times N$ sensing matrix requires maximum incoherence between $\Phi$ and $\Psi$, which is defined by

$$
\mu(\boldsymbol{\Phi}, \boldsymbol{\Psi})=\sqrt{N} \cdot \max _{1 \leq i, j \leq N}\left|\left\langle\phi_{i}, \psi_{j}\right\rangle\right|
$$

where $\phi_{i}$ is the $i^{\text {th }}$ row of $\boldsymbol{\Phi}$, and $\psi_{j}$ is the $j^{\text {th }}$ column of $\boldsymbol{\Psi}$ and $\langle\cdot\rangle$ denotes the dot product. The most used matrices are random matrices with i.i.d. entries formed by sampling a Gaussian distribution $(0,1 / \mathrm{M})$ or a symmetric Bernouilli distribution $P\left(\phi_{i, j}= \pm 1 / \sqrt{M}\right)=0.5$. However, they are not suitable for real-time mobile telemedicine applications as they are energy intensive and time consuming. The sparse binary sensing matrix (SBSM) was proposed in [16], containing $d$ non-zero entries of values $1 / \sqrt{d}$ in each column. In addition, the minimum value of $d$ can be found experimentally and is usually much smaller than $M$. For the sake of computational simplicity and energy conservation, SBSM is used for the mobile telemedicine system in this paper.

When the measurement $\mathbf{y}$ is inaccurate and corrupted with noise, (2) becomes

$$
\mathbf{y}=\boldsymbol{\Phi} \mathbf{x}+\mathbf{e}
$$

where $\mathbf{e}$ is an error term with bounded energy, i.e., $\|\mathbf{e}\|_{2} \leq \varepsilon$. These inaccurate measurements lead to a noise-aware reconstruction of $\mathbf{x}$ as an optimization problem:

$$
\hat{\mathbf{x}}=\boldsymbol{\Psi} \hat{\mathbf{s}} \text {, where } \hat{\mathbf{s}}=\min \|\mathbf{s}\|_{1} \text { subject to }\|\mathbf{y}-\boldsymbol{\Theta} \mathbf{s}\|_{2} \leq \varepsilon
$$

Reconstruction of $\mathbf{x}$ requires a proper design of sparsifying dictionary $\Psi$. It was shown that EEG signals are sparse in the Gabor domain [4], and a Gabor dictionary with optimized joint time-frequency resolution is adopted here. The atoms in such a dictionary are given by

$$
g_{\left(n, n_{0}, f_{0}, \sigma\right)}=K_{\left(n_{0}, f_{0}, \sigma\right)} \exp \left(-\frac{\left(n-n_{0}\right)^{2}}{2 \sigma^{2}}\right) \sin \left(2 \pi f_{0}\left(n-n_{0}\right)\right)(6)
$$

where $n_{0}$ and $f_{0}$ are the time and frequency centers of the atom, respectively. $\sigma$ is the spread of the atom, and $K_{\left(n_{0}, f_{0}, \sigma\right)}$ is a constant such that $\left\|g_{\left(n, n_{0}, f_{0}, \sigma\right)}\right\|_{1}=1$.

There are several algorithms available to solve the underdetermined linear system in (5). At the same level of reconstruction accuracy, studies showed that convex optimization algorithms require smaller $M$ as compared to a greedy algorithm at the expense of slightly longer convergence time [9]. We chose to use the basis pursuit denoising (BPDN) algorithm implemented in the $\mathrm{SPGL}_{1}$ Matlab solver [17] in this paper.

\section{EFFECT OF EPOCH LENGTH}

As aforementioned, the signal $\mathbf{x}$ considered in the discussion of CS in Section II is a $N \times 1$ column vector. When $\mathbf{x}$ is an epoch of EEG signal, $N$ is related to two parameters - sampling frequency $\left(f_{s}\right)$ and epoch length in seconds, which is denoted as $\mathrm{T}_{\text {epoch. }}$ Mathematically,

$$
N=f_{s} T_{\text {epoch }}
$$

When $f_{s}$ is fixed, as in most EEG recording systems, $N$ is

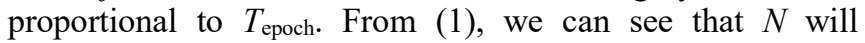
determine the size of the sparsifying dictionary. Intuitively, for Gabor dictionary, a larger $N$ means a larger number of atoms. 
A larger dictionary size will take the SPGL1 solver more time to reconstruct $\mathbf{x}$. On the contrary, a smaller $N$ will allow for a smaller Gabor dictionary size and thus a shorter signal reconstruction time. Will a short epoch length favor the performance of $C S$ in compressing EEG? This question motivates us to study the effect of epoch length on CS.

If we take the compression ratio $(\mathrm{CR})$ as another performance metric, which is defined by

$$
\mathrm{CR}=N / M
$$

We can see that $N$ also affects CR. For the sake of compression, $N$ should not be less than $M$. Therefore, a smaller $N$ will lead to a smaller CR. For mobile telemedicine, a smaller CR means more EEG data bits must be transferred over the network, and leads to a longer transmission time.

\section{Computational Methods}

In this section, we discuss the method to evaluate the effect of epoch length on the performance of CS in EEG compression. The adopted mobile telemedicine system is illustrated in Fig. 1. At the patient side, with the help of a mobile telemedicine unit, the continuous signal from EEG electrodes is first sampled at a fixed frequency $\left(\mathrm{f}_{\mathrm{s}}\right)$, and then it is divided into non-overlapped segments with equal lengths of $N$ data points. This operation is usually referred to as EEG epoching. In this paper, $N$ is a variable related to $T_{\text {epoch }}$ according to (7). In the literature, different values of $N$ such as 256 or 512 were used without any further explanation. Next, the mean of each EEG channel is removed for the sake of better signal compression. Note that the mean of each EEG epoch should be transmitted along with the EEG measurement y. Signal compression happens when the signal passes through the CS stage, where the signal is multiplied with the sensing matrix $\Phi$. After compression, the signal is transmitted wirelessly to a sever node or directly to the Internet / cloud.

At the health professional side, the EEG measurement received via a wireless receiver or the Internet / cloud is passed to the SPGL1 server with the BPDN algorithm. The Gabor dictionary $\Psi$ is loaded into the server, and used for the reconstruction of EEG epochs. Then, the particular mean value is added back to the reconstructed signal, which is displayed to a doctor, or further analyzed to produce a report.

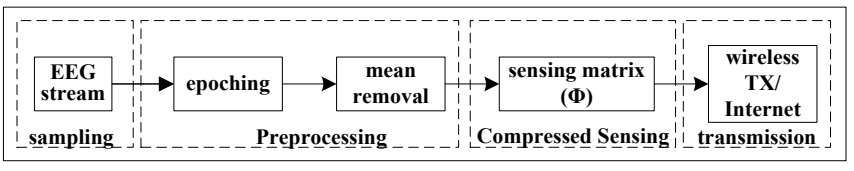

Mobile Telemedicine Unit

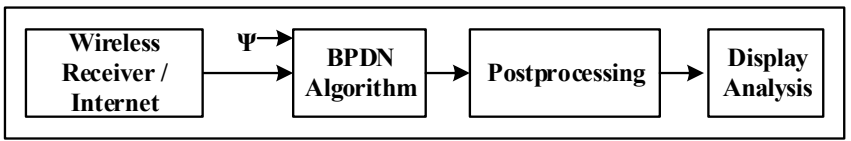

Telemedicine Station Unit

Fig. 1. Schematic of a mobile telemedicine system for EEG.

The parameter combination $\left(n_{0}, f_{0}, \sigma\right)$ controls the position and shape of a Gabor atom, which is built from translations and modulations of a generating function, as proposed by Dennis Gabor in 1946 [19]. Thus, it is important to determine how to increment each of them so that optimal time-frequency resolution is achieved in the resultant Gabor dictionary. The chosen discretization of $\sigma$ is $2^{j}$, where $0 \leq j \leq \log _{2} N$. Then, the time step size $\left(\Delta n_{0}\right)$ is proportional to $\sigma$ and the frequency step size $\left(\Delta f_{0}\right)$ is inversely proportional to $\sigma$ [18]. In particular, the following equations are used to generate $\Delta n_{0}$ and $\Delta f_{0}$ :

$$
\left\{\begin{array}{l}
\Delta n_{0}=\sigma \sqrt{C} \\
\Delta f_{0}=\frac{2 \pi \sqrt{C}}{\sigma}, \text { where } C=\frac{1}{\pi} \ln \left(\frac{1+a^{2}}{2 a}\right)
\end{array}\right.
$$

where $a$ is the ratio of neighbor spreads and chosen as 2 in this paper following [19]. Furthermore, once the value of $N$ is determined, $n_{0}$ and $f_{0}$ are confined as $0 \leq n_{0} \leq N$ and $0 \leq f_{0} \leq 0.5$.

We use the EEG data from the CHB-MIT Scalp EEG database [20] in PhysioBank [11], which are widely used to evaluate the performance of current approaches in the last two decades. The database was collected at the Children's Hospital Boston from 24 paediatric subjects with intractable seizures. Data were collected at $f_{s}=256 \mathrm{~Hz}$, with a resolution 16 bits per sample and 23 channels for several days. A desktop computer with Intel i7-6700 dual-core CPU @3.4 GHz/3.41 GHz, 32 GB of RAM, operating system 64-bit Windows 10, Matlab version R2015b (Build 8.6.0.267246) with academic license was used for the signal reconstruction.

Besides the compression ratio defined in (8), the following performance metrics are also considered: (i) normalized mean square error (NMSE), (ii) size of the Gabor dictionary in kilobytes (KB) (iii) reconstruction time [6]. First, NMSE is used to check the reconstruction quality, which is given by

$$
N M S E=\frac{\|\mathbf{x}-\hat{\mathbf{x}}\|^{2}}{\left\|\mathbf{x}-\boldsymbol{\mu}_{\mathbf{x}}\right\|^{2}}
$$

where $\mathbf{x}$ and $\hat{\mathbf{x}}$ are the original signal and the reconstructed signal, respectively. $\boldsymbol{\mu}_{\mathbf{x}}$ is the mean of $\mathbf{x}$. NMSE is used to eliminate the bias due to differences in means among different EEG epochs. The lower the NMSE value, the better the signal reconstruction.

Next, it is assumed that the Gabor dictionary is generated and loaded in the computer before it runs the SPGL1 Matlab sever. The rationale behind this assumption is that generation of a Gabor dictionary usually takes a while, especially when $N$ is large. In addition, when a fixed Gabor dictionary is used, it is time-efficient to load the dictionary into the random-access memory (RAM) of a computer. The size of the Gabor dictionary indicates the amount of disk space required to store it, as well as the amount of time to load it to the RAM.

Finally, reconstruction time refers to the amount of CPU time for an algorithm to find the reconstructed signal ( $\hat{\mathbf{x}})$ from a measurement $(\mathbf{y})$ of its original signal $(\mathbf{x})$.

\section{RESUlTS AND Discussion}

The value of $T_{\text {epoch }}$ is varied from $1 \mathrm{~s}$ to $2 \mathrm{~s}$, then $4 \mathrm{~s}$ and finally $8 \mathrm{~s}$. We do not perform any resampling and $f_{s}$ is fixed at $256 \mathrm{~Hz}$ as originally recorded. At each $T_{\text {epoch }}$ setting, $100 \mathrm{EEG}$ epochs are generated with a random starting point and a length of $N$ data points from an arbitrary channel in any of the 24 recordings, and passed to our CS Matlab program with the 
SPGL1 solver. There are some dead channels in which the signal values are zeros. However, unlike [9], they are not removed to enable us to simulate a practical EEG test that occasionally has loose electrodes.

Fig. 2 shows the effect of epoch length on NMSE at different $C R$ values. Larger $T_{\text {epoch }}$ results in better signal compression with a lower NMSE. Fig. 3 shows the average reconstruction time for an EEG epoch when the epoch length is increased at different $\mathrm{CR}$ values. The longer the epoch length, the longer the reconstruction time. It is interesting to see that at a fixed epoch length, the larger the $\mathrm{CR}$ value, the shorter the signal reconstruction time. This is due to the fact that when CR is large, the size of $\mathbf{y}$ becomes smaller and it reduces the computational complexity of (5). Next, Table I summarizes the effect of epoch length on the number of atoms used in the Gabor dictionary, and on the memory space required to store such a dictionary.

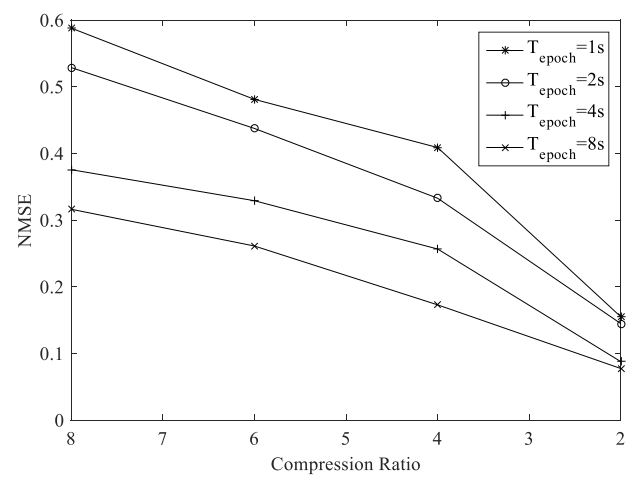

Fig. 2 Effect of epoch length on NMSE

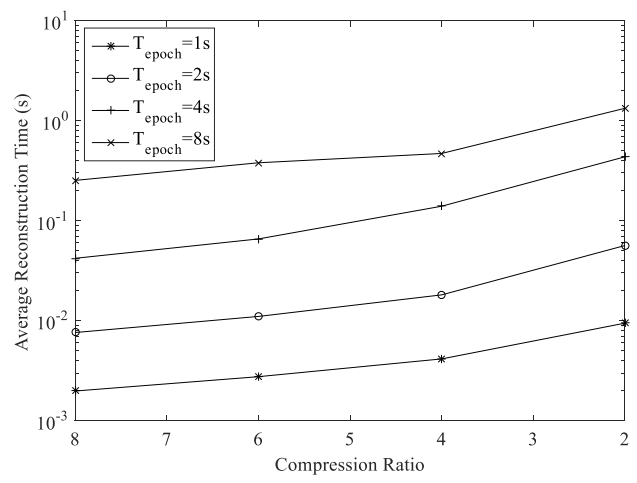

Fig. 3. Effect of epoch length on reconstruction time

TABLE I. EFFECT OF EPOCH LENGTH ON GABOR DiCTIONARY SiZE

\begin{tabular}{|c|c|c|}
\hline $\mathbf{T}_{\text {epoch }}(\mathbf{s})$ & Gabor Dictionary Size $^{*}(\mathrm{kB})$ & Number of Atoms \\
\hline 1 & 10,293 & 2,573 \\
\hline 2 & 40,842 & 5,105 \\
\hline 4 & 162,099 & 10,131 \\
\hline 8 & 645,893 & 20,184 \\
\hline
\end{tabular}

This paper investigated the effect of epoch length on the performance of compressed sensing in compressing EEG signals. When a Gabor dictionary is used, epoch length together with the sampling frequency will directly determine the size of the Gabor dictionary. Experimental results (with sampling frequency of $256 \mathrm{~Hz}$ ) suggest that a suitable epoch length is $4 \mathrm{~s}$, which might not be the optimal one, as the rigid analytical model is yet to be formulated. It is worthy studying how to use an adaptive epoch length when a dynamic Gabor dictionary is used for compressed sensing of EEG, which is left as our future work on this topic.

\section{REFERENCES}

[1] Z. Zhang, T.-P. Jung, S. Makeig, and B. D. Rao, "Compressed sensing of EEG for wireless telemonitoring with low energy consumption and inexpensive hardware," IEEE Transactions on Biomedical Engineering, vol. 60, pp. 221-224, 2013.

[2] G. Antoniol and P. Tonella, "EEG data compression techniques," IEEE Transactions on Biomedical Engineering, vol. 44, pp. 105-114, 1997.

[3] D. L. Donoho, "Compressed sensing," IEEE Transactions on Information Theory, vol. 52, pp. 1289-1306, April 2006.

[4] S. Aviyente, "Compressed sensing framework for EEG compression," in Proc. of IEEE SSP '07, Madison, WI, 2007, pp. 181-184.

[5] A. M. Abdulghani, A. J. Casson, and E. Rodriguez-Villegas, "Quantifying the feasibility of compressive sensing in portable electroencephalography systems," in Proc. of $5^{\text {th }}$ International Conference Foundations of Augmented Cognition, San Diego, CA, 2009, pp. 319-328.

[6] A. M. Abdulghani, A. J. Casson, and E. Rodriguez-Villegas, "Compressive sensing scalp EEG signals: implementations and practical performance," Medical Biological Engineering Computing, vol. 50, pp. 1137-1145, 2012.

[7] W. J. Levy, "Effect of epoch length on power spectrum analysis of the EEG," Anesthesiology, vol. 66, pp. 489-495, 1987.

[8] J. Möcks and T. Gasser, "How to select epochs of the EEG at rest for quantitative analysis," Electroencephalography and Clinical Neurophysiology, vol. 58, pp. 89-92, July 1984.

[9] S. Fauvel and R. K. Ward, "An energy efficient compressed sensing framework for the compression of electroencephalogram signals," Sensors, vol. 14, pp. 1474-1496, 2014.

[10] D. Craven, B. McGinley, L. Kilmartin, M. Glavin, and E. Jones, "Compressed sensing for bioelectric signals: A review," IEEE Journal of Biomedical and Health Informatics, vol. 19, pp. 529-540, 2015.

[11] A. L. Goldberger, et al., "PhysioBank, PhysioToolkit, and PhysioNet component of a new research resource for complex physiologic signals," Circulation Electronic Pages, 2002.

[12] Y. Liu, M. De Vos, and S. Huffel, "Compressed sensing of multi-channel EEG signals: the simultaneous cosparsity and low rank optimization," IEEE Transactions on Biomedical Engineering, vol. PP, pp. 2055-2061, August 2015.

[13] B. Allison, E. Wolpaw, and J. Wolpaw, "Brain computer interface systems: Progress and prospects," Expert Review of Medical Devices, vol. 4, pp. 463-474, July 2007.

[14] S. Sanei and J. A. Chambers, EEG Signal Processing: John Wiley \& Sons, 2007.

[15] C. A. Marsan and L. S. Zivin, "Factors related to the occurrence of typical paroxysmal abnormalities in the EEG records of epileptic patients," Epilepsia, vol. 11, pp. 361-381, 1970.

[16] H. Mamaghanian, N. Khaled, D. Atienza, and P. Vandergheynst, "Compressed sensing for real-time energy-efficient ECG compression on wireless body sensor nodes," IEEE Transactions on Biomedical Engineering, vol. 58, pp. 2456-2466, 2011.

[17] E. v. d. Berg and M. P. Friedlander. (2007). SPGL1: A solver for large-scale sparse reconstruction. Available: http://www.cs.ubc.ca/labs/scl/spgl1

[18] P. J. Durka, "Time-frequency analyses of EEG," PhD, Department of Physics, Warsaw University, Poland, 1996.

[19] M. Barwinski, "Product-based metric for Gabor functions and its implications for the matching pursuit algorithm," MSc, Faculty of Physics, Warsaw University, Poland, 2004.

[20] A. H. Shoeb, "Application of Machine Learning to Epileptic Seizure Onset Detection and Treatment," Harvard-MIT Division of Health Sciences and Technology, Massachusetts Institute of Technology, USA, 2009. 